ANNALES

POLONICI MATHEMATICI

$84.1(2004)$

\title{
Product preserving bundles on foliated manifolds
}

\author{
by WŁodzimierz M. Mikulski (Kraków)
}

\begin{abstract}
We present a complete description of all product preserving bundle functors on the category $\mathcal{F}$ ol of all foliated manifolds and their leaf respecting maps in terms of homomorphisms of Weil algebras.
\end{abstract}

Introduction. Product preserving bundle functors on the category $\mathcal{M f}$ of manifolds and maps play an important role in modern differential geometry. To such bundle functors one can lift some geometric structures, like vector fields, forms, connections, etc. To define such lifts, only the product preserving property of such functors is important. Such functors have been classified by means of Weil algebras [2].

Similar research has been done on fibred manifolds. The class of product preserving functors is a wide class of bundle functors on the category $\mathcal{F} \mathcal{M}$ of fibred manifolds and their fibre respecting maps. Such functors have been classified by means of homomorphisms of Weil algebras [3] (see also [1] for a simpler proof).

The class of product preserving functors is a wide class of bundle functors on the category $\mathcal{F}$ ol of foliated manifolds without singularities and their leaf respecting maps. For example, the normal bundle functor $N: \mathcal{F}$ ol $\rightarrow \mathcal{F} \mathcal{M}$ sending a foliated manifold $(M, \mathcal{F})$ to its normal bundle $N(M, \mathcal{F})$ and a leaf respecting map $f:\left(M_{1}, \mathcal{F}_{1}\right) \rightarrow\left(M_{2}, \mathcal{F}_{2}\right)$ to the induced map $N(f)$ : $N\left(M_{1}, \mathcal{F}_{1}\right) \rightarrow N\left(M_{2}, \mathcal{F}_{2}\right)$ is product preserving. More generally, for any Weil algebra $A$ the bundle functor $A: \mathcal{F}$ ol $\rightarrow \mathcal{F} \mathcal{M}$ of transverse $A$-points sending a foliated manifold $(M, \mathcal{F})$ to the bundle $A(M, \mathcal{F})$ of transverse $A$-points in the sense of R. Wolak [5] and a leaf respecting map $f:\left(M_{1}, \mathcal{F}_{1}\right) \rightarrow\left(M_{2}, \mathcal{F}_{2}\right)$ to the induced map $A(f): A\left(M_{1}, \mathcal{F}_{1}\right) \rightarrow A\left(M_{2}, \mathcal{F}_{2}\right)$ is product preserving. Also, the usual Weil bundle functor $T^{A}: \mathcal{M} f \rightarrow \mathcal{F} \mathcal{M}$ on manifolds can

2000 Mathematics Subject Classification: 58A05, 58A20, 57R30.

Key words and phrases: (product preserving) bundle functors, natural transformations, Weil algebras, foliated manifolds. 
be considered as the product preserving bundle functor $T^{A}: \mathcal{F}_{o l} \rightarrow \mathcal{F M}$ satisfying $T^{A}(M, \mathcal{F})=T^{A} M$ for any foliated manifold $(M, \mathcal{F})$.

So, it seems natural and useful to classify all product preserving bundle functors on the category $\mathcal{F}$ ol. This is the purpose of the present note. The main results can be stated as follows:

TheOREM A. There is a bijection between the isomorphism classes of product preserving bundle functors on $\mathcal{F}$ ol and the isomorphism classes of Weil algebra homomorphisms.

THEOREM B. Given two product preserving bundle functors on $\mathcal{F}$ ol there is a bijection between their natural transformations and morphisms of the corresponding Weil algebra homomorphisms.

The category $\mathcal{F} \mathcal{M}$ of fibred manifolds is in an obvious way a subcategory of $\mathcal{F}$ ol (we treat a fibred manifold as a manifold with foliation given by the fibres). Therefore any product preserving bundle functor $F: \mathcal{F}$ ol $\rightarrow \mathcal{F} \mathcal{M}$ can be restricted to a product preserving bundle functor $F: \mathcal{F M} \rightarrow \mathcal{F} \mathcal{M}$. Then by the classifying result from [3] we have the corresponding homomorphism $\mu^{F}: A^{F} \rightarrow B^{F}$ of Weil algebras. Conversely, any Weil algebra homomorphism $\mu: A \rightarrow B$ induces a product preserving bundle functor $T^{\mu}: \mathcal{F M} \rightarrow \mathcal{F} \mathcal{M}$ (see [3]). Since any foliated manifold is locally a fibred manifold, the bundle functor $T^{\mu}: \mathcal{F} \mathcal{M} \rightarrow \mathcal{F} \mathcal{M}$ can be extended (by gluing) to $T^{\mu}: \mathcal{F}$ ol $\rightarrow \mathcal{F} \mathcal{M}$. However, the gluing method is rather inconvenient. That is why we will present another strictly canonical construction of $T^{\mu}: \mathcal{F}$ ol $\rightarrow \mathcal{F} \mathcal{M}$.

All manifolds are assumed to be finite-dimensional. All manifolds and maps are assumed to be smooth, i.e. of class $\mathcal{C}^{\infty}$. All foliations are assumed to be without singularities.

1. Basic definitions. Let us recall the following definitions (see e.g. [2]). Let $F: \mathcal{F}$ ol $\rightarrow \mathcal{F} \mathcal{M}$ be a covariant functor. Let $B_{\mathcal{F} \mathcal{M}}: \mathcal{F} \mathcal{M} \rightarrow \mathcal{M f}$ be the base functor and $B_{\mathcal{F}_{o l}}: \mathcal{F}_{o l} \rightarrow \mathcal{M f}$ be the forgetful functor.

$A$ bundle functor on $\mathcal{F}$ ol is a functor $F$ as above satisfying:

(i) (Base preservation) $B_{\mathcal{F} \mathcal{M}} \circ F=B_{\mathcal{F} o l}$. Hence the induced projections form a functor transformation $\pi: F \rightarrow B_{\mathcal{F}_{\text {ol }}}$.

(ii) (Localization) For every inclusion $i_{(U, \mathcal{F} \mid U)}:(U, \mathcal{F} \mid U) \rightarrow(M, \mathcal{F})$ of an open subset, $F(U, \mathcal{F} \mid U)$ is the restriction $\pi^{-1}(U)$ of $\pi: F(M, \mathcal{F})$ $\rightarrow M$ over $U$ and $F i_{(U, \mathcal{F} \mid U)}$ is the inclusion $\pi^{-1}(U) \rightarrow F(M, \mathcal{F})$.

Given two bundle functors $F_{1}, F_{2}$ on $\mathcal{F}$ ol, by a natural transformation $\nu: F_{1} \rightarrow F_{2}$ we shall mean a system of base preserving fibred maps $\nu$ : $F_{1}(M, \mathcal{F}) \rightarrow F_{2}(M, \mathcal{F})$ for every foliated manifold $(M, \mathcal{F})$ satisfying the condition $F_{2} f \circ \nu=\nu \circ F_{1} f$ for every $\mathcal{F}_{\text {ol-morphism } f \text {. }}$. 
A bundle functor $F$ on $\mathcal{F}_{o l}$ is product preserving if for any product projections $\left(M_{1}, \mathcal{F}_{1}\right) \stackrel{\mathrm{pr}_{1}}{\longleftarrow}\left(M_{1}, \mathcal{F}_{1}\right) \times\left(M_{2}, \mathcal{F}_{2}\right) \stackrel{\mathrm{pr}_{2}}{\longrightarrow}\left(M_{2}, \mathcal{F}_{2}\right)$ (in the category $\mathcal{F}$ ol $), F\left(M_{1}, \mathcal{F}_{1}\right) \stackrel{F \mathrm{pr}_{1}}{\longleftarrow} F\left(\left(M_{1}, \mathcal{F}_{1}\right) \times\left(M_{2}, \mathcal{F}_{2}\right)\right) \stackrel{F \mathrm{pr}_{2}}{\longrightarrow} F\left(M_{2}, \mathcal{F}_{2}\right)$ are product projections in the category $\mathcal{F} \mathcal{M}$. In other words, $F\left(\left(M_{1}, \mathcal{F}_{1}\right) \times\left(M_{2}, \mathcal{F}_{2}\right)\right)=$ $F\left(M_{1}, \mathcal{F}_{1}\right) \times F\left(M_{2}, \mathcal{F}_{2}\right)$ modulo $\left(F \operatorname{pr}_{1}, F \mathrm{pr}_{2}\right)$.

Some known examples of product preserving bundle functors on $\mathcal{F}$ ol have been mentioned in the introduction.

\section{Product preserving bundle functors on $\mathcal{F}_{o l}$ corresponding to} Weil algebra homomorphisms. Let $A$ be an associative algebra over the field $\mathbb{R}$ with unit 1 . The algebra $A$ is called a Weil algebra if it is commutative, of finite dimension over $\mathbb{R}$, and if it has a unique maximal ideal $\underline{A}$ of codimension 1 such that $\underline{A}^{h+1}=0$ for some non-negative integer $h$.

Let $\mu: A \rightarrow B$ be a homomorphism of Weil algebras. We are going to construct a product preserving bundle functor $T^{\mu}: \mathcal{F}$ ol $\rightarrow \mathcal{F} \mathcal{M}$.

Example 1. For a foliated manifold $(M, \mathcal{F})$ and $x \in M$ we denote by $C_{x}^{\infty}(M)$ the algebra of germs at $x$ of smooth maps $M \rightarrow \mathbb{R}$, and by $C_{x}^{\infty}(M, \mathcal{F})$ the subalgebra of maps constant on the fibres. Let $T_{x}^{\mu}(M, \mathcal{F})$ be the set of pairs $(\varphi, \psi)$ of algebra homomorphisms $\varphi: C_{x}^{\infty}(M, \mathcal{F}) \rightarrow A$ and $\psi: C_{x}^{\infty}(M) \rightarrow B$ such that

$$
\psi(u v)=\mu(\varphi(u)) \psi(v)
$$

for any $u \in C_{x}^{\infty}(M, \mathcal{F})$ and $v \in C_{x}^{\infty}(M)$. Put $T^{\mu}(M, \mathcal{F})=\bigcup_{x \in M} T_{x}^{\mu}(M, \mathcal{F})$. Then the obvious projection $\pi: T^{\mu}(M, \mathcal{F}) \rightarrow M$ is a smooth bundle. More precisely, given adapted coordinates $\left(x^{1}, \ldots, x^{q}, y^{1}, \ldots, y^{p}\right)$ on $(M, \mathcal{F})$, where $\mathcal{F}$ is $p$-dimensional and $M$ is $p+q$-dimensional, we have the induced coordinates $\left(\widetilde{x}^{1}, \ldots, \widetilde{x}^{q}, \widetilde{y}^{1}, \ldots, \widetilde{y}^{p}\right): T^{\mu}(M, \mathcal{F}) \mid U \rightarrow A^{q} \times B^{p}$ such that $\widetilde{x}^{i}(\varphi)=\varphi\left(\left[x^{i}\right]_{x}\right) \in A$ and $\widetilde{y}^{j}(\psi)=\psi\left(\left[y^{j}\right]_{x}\right) \in B$ for any $(\varphi, \psi) \in T_{x}^{\mu}(M, \mathcal{F})$, $x \in M$. (Condition $(*)$ implies that $(\varphi, \psi) \in T_{x}^{\mu}(M, \mathcal{F})$ is uniquely determined by its induced coordinates.)

Every $\mathcal{F}_{\text {ol-map }} f:\left(M_{1}, \mathcal{F}_{1}\right) \rightarrow\left(M_{2}, \mathcal{F}_{2}\right)$ induces a fibred map $T^{\mu} f:$ $T^{\mu}\left(M_{1}, \mathcal{F}_{1}\right) \rightarrow T^{\mu}\left(M_{2}, \mathcal{F}_{2}\right)$ covering $f$ such that $T^{\mu} f(\varphi, \psi)=(\bar{\varphi}, \bar{\psi})$ for any $(\varphi, \psi) \in T_{x}^{\mu}\left(M_{1}, \mathcal{F}_{1}\right)$ and $x \in M_{1}$, where $\bar{\varphi}: C_{f(x)}^{\infty}\left(M_{2}, \mathcal{F}_{2}\right) \rightarrow A$ is defined by $\bar{\varphi}(u)=\varphi(u \circ f)$ and $\bar{\psi}: C_{f(x)}^{\infty}\left(M_{2}\right) \rightarrow B$ is defined by $\bar{\psi}(v)=\psi(v \circ f)$ for $u \in C_{f(x)}^{\infty}\left(M_{2}, \mathcal{F}_{2}\right)$ and $v \in C_{f(x)}^{\infty}(M)$.

If in adapted coordinates a foliated map is of the form $f: \mathbb{R}^{q} \times \mathbb{R}^{p} \rightarrow \mathbb{R}^{\bar{q}} \times$ $\mathbb{R}^{\bar{p}}, f\left(x_{i}, y_{j}\right)=\left(f_{1}\left(x_{i}\right), f_{2}\left(x_{i}, y_{j}\right)\right)$ for some $f_{1}: \mathbb{R}^{q} \rightarrow \mathbb{R}^{\bar{q}}$ and $f_{2}: \mathbb{R}^{q} \times \mathbb{R}^{p} \rightarrow$ $\mathbb{R}^{\bar{p}}$, then in the corresponding induced coordinates $T^{\mu} f: A^{q} \times B^{p} \rightarrow A^{\bar{q}} \times B^{\bar{p}}$, $T^{\mu} f\left(a_{i}, b_{j}\right)=\left(T_{A} f_{1}\left(a_{i}\right), T_{B} f_{2}\left(\mu\left(a_{i}\right), b_{j}\right)\right)$, where $T_{A}: \mathcal{M} f \rightarrow \mathcal{F} \mathcal{M}$ is the Weil functor [2]. 
The correspondence $T^{\mu}: \mathcal{F}_{o l} \rightarrow \mathcal{F} \mathcal{M}$ is a product preserving bundle functor. It is called the product preserving bundle functor on $\mathcal{F}$ ol corresponding to the Weil algebra homomorphism $\mu$.

REMARK 1. Observe that given a $p$-dimensional foliation $\mathcal{F}$ on a $p+q$ dimensional manifold $M$ the bundle $T^{\mu}(M, \mathcal{F})$ admits a canonical $p \operatorname{dim}_{\mathbb{R}} B$ dimensional foliation $\mathcal{F}^{\mu}$. In the induced coordinates the leaves of $\mathcal{F}^{\mu}$ are of the form $\{a\} \times B^{p} \subset A^{q} \times B^{p}$.

\section{Weil algebra homomorphisms corresponding to product pre-} serving bundle functors on $\mathcal{F}$ ol. Let $F: \mathcal{F} o l \rightarrow \mathcal{F} \mathcal{M}$ be a product preserving bundle functor. We are going to construct a Weil algebra homomorphism $\mu^{F}: A^{F} \rightarrow B^{F}$.

Example 2. We define $\mu^{F}: A^{F} \rightarrow B^{F}$ to be the Weil algebra homomorphism corresponding to the product preserving bundle functor $F_{\mid \mathcal{F M}}$ : $\mathcal{F} \mathcal{M} \rightarrow \mathcal{F} \mathcal{M}$ (see [3]). We call it the Weil algebra homomorphism corresponding to the product preserving bundle functor $F$ on $\mathcal{F}_{o l}$.

More precisely, we put $A^{F}=F\left(\mathbb{R}, \mathcal{F}^{\prime}\right)$ and $B^{F}=F\left(\mathbb{R}, \mathcal{F}^{\prime \prime}\right)$, where $\mathcal{F}^{\prime}$ is the 0 -dimensional foliation on $\mathbb{R}$ and $\mathcal{F}^{\prime \prime}$ is the foliation on $\mathbb{R}$ with one leaf $\mathbb{R}$. The sum mappings are given by $+_{A^{F}}=F(+): A^{F} \times A^{F} \rightarrow A^{F}$ and $+_{B^{F}}=F(+): B^{F} \times B^{F} \rightarrow B^{F}$, where $+: \mathbb{R} \times \mathbb{R} \rightarrow \mathbb{R}$ is the sum map considered as the corresponding $\mathcal{F}$ ol-morphisms. Similarly, the multiplications of $A^{F}$ and $B^{F}$ are obtained by applying $F$ to the multiplication of $\mathbb{R}$ viewed as the corresponding $\mathcal{F}$ ol-morphisms. The zero maps and the unity maps are obtained by applying $F$ to the zero and the unity map of $\mathbb{R}$. The homomorphism $\mu^{F}: A^{F} \rightarrow B^{F}$ is $F\left(\mathrm{id}_{\mathbb{R}}\right): F\left(\mathbb{R}, \mathcal{F}^{\prime}\right) \rightarrow$ $F\left(\mathbb{R}, \mathcal{F}^{\prime \prime}\right)$, where $\mathrm{id}_{\mathbb{R}}$ is the identity map of $\mathbb{R}$ treated as the corresponding $\mathcal{F}$ ol-morphism.

The following facts will not be used later.

FACT 1. The Weil algebra homomorphism corresponding to the normal bundle functor $N: \mathcal{F}$ ol $\rightarrow \mathcal{F M}$ (see the introduction) is the unique algebra homomorphism $\kappa_{\mathbb{D}}: \mathbb{D} \rightarrow \mathbb{R}$, where $\mathbb{D}$ is the Weil algebra of dual numbers.

FACT 2. The Weil algebra homomorphism corresponding to the bundle functor $A: \mathcal{F}$ ol $\rightarrow \mathcal{F} \mathcal{M}$ of transverse A-points (see the introduction) is the unique algebra homomorphism $\kappa_{A}: A \rightarrow \mathbb{R}$.

FACT 3. The Weil algebra homomorphism corresponding to the product preserving bundle functor $T^{A}: \mathcal{F}$ ol $\rightarrow \mathcal{F} \mathcal{M}$ of $A$-near points (see the introduction) is the identity $\operatorname{map} \operatorname{id}_{A}: A \rightarrow A$. 
4. Classification of product preserving bundle functors on $\mathcal{F}_{o l}$ in terms of Weil algebra homomorphisms. The following proposition shows that any product preserving bundle functor on $\mathcal{F}$ ol is equivalent to some product preserving bundle functor as in Example 1.

Proposition 1. Let $F: \mathcal{F}$ ol $\rightarrow \mathcal{F} \mathcal{M}$ be a product preserving bundle functor. Let $\mu^{F}: A^{F} \rightarrow B^{F}$ be the corresponding Weil algebra homomorphism. Then we have a natural equivalence $\Theta^{F}: F \cong T^{\mu^{F}}$.

Proof. Let $(M, \mathcal{F})$ be a $\mathcal{F}$ ol-object. We construct canonically a diffeomorphism $\Theta^{F}: F(M, \mathcal{F}) \rightarrow T^{\mu^{F}}(M, \mathcal{F})$ as follows. Given a point $y \in$ $F_{x}(M, \mathcal{F}), x \in M$, we define $\Theta^{F}(y)=\left(\varphi_{y}, \psi_{y}\right)$, where $\varphi_{y}: C_{x}^{\infty}(M, \mathcal{F}) \rightarrow$ $A^{F}, \psi_{y}: C_{x}^{\infty}(M) \rightarrow B^{F}, \varphi_{y}\left([f]_{x}\right)=F f(y), \psi_{y}\left([g]_{x}\right)=F g(y)$ for $[f]_{x} \in$ $C_{x}^{\infty}(M, \mathcal{F})$ and $[g]_{x} \in C_{x}^{\infty}(M)$, where $f$ and $g$ are treated as the corresponding $\mathcal{F}$ ol-morphisms. Recalling the definition of $\mu^{F}: A^{F} \rightarrow B^{F}$ (Example 2) and using the functoriality of $F$ one can standardly verify that $\Theta^{F}(y) \in T_{x}^{\mu^{F}}(M, \mathcal{F})$.

It remains to show that $\Theta^{F}: F(M, \mathcal{F}) \rightarrow T^{\mu^{F}}(M, \mathcal{F})$ is a diffeomorphism.

Because $\Theta^{F}: F \rightarrow T^{\mu^{F}}$ is natural with respect to $\mathcal{F}$ ol-maps and $F$ and $T^{\mu^{F}}$ preserve products and $(M, \mathcal{F})$ is locally a (multi)product of $\left(\mathbb{R}, \mathcal{F}^{\prime}\right)$ and $\left(\mathbb{R}, \mathcal{F}^{\prime \prime}\right)$ we may assume that $(M, \mathcal{F})=\left(\mathbb{R}, \mathcal{F}^{\prime}\right)$ or $(M, \mathcal{F})=\left(\mathbb{R}, \mathcal{F}^{\prime \prime}\right)$, the trivial foliations on $\mathbb{R}$. But for $(M, \mathcal{F})=\left(\mathbb{R}, \mathcal{F}^{\prime}\right)$ the transformation $\Theta^{F}$ is the composition $F\left(\mathbb{R}, \mathcal{F}^{\prime}\right)=A^{F}=T^{\mu^{F}}\left(\mathbb{R}, \mathcal{F}^{\prime}\right)$, where the second equality is given by the induced coordinate $\widetilde{x}$ (see Example 1). Similarly, for $(M, \mathcal{F}$ ) $=\left(\mathbb{R}, \mathcal{F}^{\prime \prime}\right)$ the transformation $\Theta^{F}$ is the composition $F\left(\mathbb{R}, \mathcal{F}^{\prime \prime}\right)=B^{F}=$ $T^{\mu}\left(\mathbb{R}, \mathcal{F}^{\prime \prime}\right)$, where the second equality is given by the induced coordinate $\widetilde{y}$ (see Example 1).

REMARK 2. Let $F: \mathcal{F}$ ol $\rightarrow \mathcal{F} \mathcal{M}$ be a product preserving bundle functor. By Proposition 1 we have a canonical diffeomorphism $\Theta^{F}: F(M, \mathcal{F}) \rightarrow$ $T^{\mu^{F}}(M, \mathcal{F})$ for any foliated manifold $(M, \mathcal{F})$. As we know, $T^{\mu^{F}}(M, \mathcal{F})$ admits the foliation $\mathcal{F}^{\mu^{F}}$ (see Remark 1). Then $F(M, \mathcal{F})$ admits the foliation $\mathcal{F}^{F}=\left(\Theta^{F}\right)^{-1} \mathcal{F}^{\mu^{F}}$. Hence $F$ has values in the category $\mathcal{F}_{\text {ol }}$. Therefore we can compose two product preserving bundle functors on $\mathcal{F}$ ol. This composition is again a product preserving bundle functor on $\mathcal{F}$ ol.

5. Classification of Weil algebra homomorphisms in terms of product preserving bundle functors on $\mathcal{F}$ ol. The following proposition shows that any Weil algebra homomorphism is isomorphic to the Weil algebra homomorphism corresponding to some product preserving bundle functor on $\mathcal{F}$ ol. 
Proposition 2. Let $\mu: A \rightarrow B$ be a Weil algebra homomorphism. Let $F=T^{\mu}$. Then we have an isomorphism $\mathcal{O}^{\mu}: \mu \cong \mu^{F}$ of Weil algebra homomorphisms.

We recall that a morphism $\mu_{1} \rightarrow \mu_{2}$ of Weil algebra homomorphisms $\mu_{1}: A_{1} \rightarrow B_{1}$ and $\mu_{2}: A_{2} \rightarrow B_{2}$ is a pair $\eta$ of Weil algebra homomorphisms $\eta_{1}: A_{1} \rightarrow A_{2}$ and $\eta_{2}: B_{1} \rightarrow B_{2}$ such that $\eta_{2} \circ \mu_{1}=\mu_{2} \circ \eta_{1}$.

Proof. We define $\mathcal{O}^{\mu}: \mu \rightarrow \mu^{F}$ to be the pair of Weil algebra isomorphisms $\mathcal{O}_{1}^{\mu}: A \cong T^{\mu}\left(\mathbb{R}, \mathcal{F}^{\prime}\right)=F\left(\mathbb{R}, \mathcal{F}^{\prime}\right)=A^{F}$ and $\mathcal{O}_{2}^{\mu}: B \cong T^{\mu}\left(\mathbb{R}, \mathcal{F}^{\prime \prime}\right)=$ $F\left(\mathbb{R}, \mathcal{F}^{\prime \prime}\right)=B^{F}$, where $\cong$ are the induced coordinates $\widetilde{x}$ and $\widetilde{y}$ (see Example 1) respectively.

One can show standardly that $\mathcal{O}^{\mu}$ is a morphism $\mu \rightarrow \mu^{F}$ of Weil algebra homomorphisms.

6. Natural transformations of product preserving bundle functors on $\mathcal{F} o l$ and induced morphisms between corresponding Weil algebra homomorphisms. Let $F_{1}, F_{2}: \mathcal{F}_{o l} \rightarrow \mathcal{F} \mathcal{M}$ be product preserving bundle functors. Let $\mu^{F_{1}}: A^{F_{1}} \rightarrow B^{F_{1}}$ and $\mu^{F_{2}}: A^{F_{2}} \rightarrow B^{F_{2}}$ be the corresponding Weil algebra homomorphisms. Let $\nu: F_{1} \rightarrow F_{2}$ be a natural transformation.

EXAmple 3. Define a morphism $\eta^{\nu}=\left(\eta_{1}^{\nu}, \eta_{2}^{\nu}\right): \mu^{F_{1}} \rightarrow \mu^{F_{2}}$ of Weil algebra homomorphisms by $\eta_{1}^{\nu}=\nu_{\left(\mathbb{R}, \mathcal{F}^{\prime}\right)}: A^{F_{1}} \rightarrow A^{F_{2}}$ and $\eta_{2}^{\nu}=\nu_{\left(\mathbb{R}, \mathcal{F}^{\prime \prime}\right)}$ : $B^{F_{1}} \rightarrow B^{F_{2}}$.

If $\nu$ is an isomorphism, then so is $\eta^{\nu}$.

We call $\eta^{\nu}$ the morphism of Weil algebra homomorphisms corresponding to $\nu$.

7. Morphisms between Weil algebra homomorphisms and induced natural transformations between corresponding product preserving bundle functors on $\mathcal{F}$ ol. Let $\mu_{1}: A_{1} \rightarrow B_{1}$ and $\mu_{2}: A_{2}$ $\rightarrow B_{2}$ be Weil algebra homomorphisms. Let $\eta=\left(\eta_{1}, \eta_{2}\right): \mu_{1} \rightarrow \mu_{2}$ be a morphism of Weil algebra homomorphisms.

EXAMPLE 4. Given a $\mathcal{F}$ ol-object $(M, \mathcal{F})$ define a base preserving fibred $\operatorname{map} \nu^{\eta}: T^{\mu_{1}}(M, \mathcal{F}) \rightarrow T^{\mu_{2}}(M, \mathcal{F})$ by $\nu^{\eta}(\varphi, \psi)=\left(\eta_{1} \circ \varphi, \eta_{2} \circ \psi\right)$ for $(\varphi, \psi) \in$ $T_{x}^{\mu_{1}}(M, \mathcal{F}), x \in M$. The family $\nu^{\eta}: T^{\mu_{1}} \rightarrow T^{\mu_{2}}$ is a natural transformation. If $\eta$ is an isomorphism, then so is $\nu^{\eta}$.

We call $\nu^{\eta}$ the natural transformation corresponding to $\nu$.

8. Object Classification Theorem. The first main result in this paper is the following theorem. 
THEOREM 1. The correspondence $F \rightarrow \mu^{F}$ induces a bijective correspondence between the equivalence classes of product preserving bundle functors on $\mathcal{F}$ ol and the equivalence classes of Weil algebra homomorphisms. The inverse correspondence is induced by the correspondence $\mu \rightarrow T^{\mu}$.

Proof. The correspondence $[F] \rightarrow\left[\mu^{F}\right]$ is well defined. For, if $\nu: F_{1} \rightarrow F_{2}$ is an isomorphism, then so is $\eta^{\nu}: \mu^{F_{1}} \rightarrow \mu^{F_{2}}$.

The correspondence $[\mu] \rightarrow\left[T^{\mu}\right]$ is well defined. For, if $\eta: \mu_{1} \rightarrow \mu_{2}$ is an isomorphism, then so is $\nu^{\eta}: T^{\mu_{1}} \rightarrow T^{\mu_{2}}$.

From Proposition 1 it follows that $[F]=\left[T^{\mu^{F}}\right]$. From Proposition 2 it follows that $[\mu]=\left[\mu^{F}\right]$ if $F=T^{\mu}$.

9. Morphism Classification Theorem. Let $F_{1}$ and $F_{2}$ be two product preserving bundle functors on $\mathcal{F}$ ol. Let $\mu^{F_{1}}: A^{F_{1}} \rightarrow B^{F_{1}}$ and $\mu^{F_{2}}$ : $A^{F_{2}} \rightarrow B^{F_{2}}$ be the corresponding Weil algebra homomorphisms.

Lemma 1. Let $\eta=\left(\eta_{1}, \eta_{2}\right): \mu^{F_{1}} \rightarrow \mu^{F_{2}}$ be a morphism of Weil algebra homomorphisms. Let $\nu^{[\eta]}: F_{1} \rightarrow F_{2}$ be a natural transformation given by the composition

$$
F_{1} \stackrel{\Theta^{F_{1}}}{\longrightarrow} T^{\mu^{F_{1}}} \stackrel{\nu^{\eta}}{\longrightarrow} T^{\mu^{F_{2}}} \stackrel{\left(\Theta^{F_{2}}\right)^{-1}}{\longrightarrow} F_{2},
$$

where $\Theta^{F}$ is as in Proposition 1 and $\nu^{\eta}$ is as described in Example 4. Then $\nu=\nu^{[\eta]}$ is the unique natural transformation $F_{1} \rightarrow F_{2}$ such that $\eta^{\nu}=\eta$, where $\eta^{\nu}$ is as in Example 3.

Proof. Suppose $\bar{\nu}: F_{1} \rightarrow F_{2}$ is another natural transformation such that $\eta^{\bar{\nu}}=\eta$. Then $\bar{\nu}$ coincides with $\nu$ on $\left(\mathbb{R}, \mathcal{F}^{\prime}\right)$ and $\left(\mathbb{R}, \mathcal{F}^{\prime \prime}\right)$. Hence $\bar{\nu}=\nu$ by the same argument as in the proof of Proposition 1.

Now, the following second main result of this paper is clear.

THEOREM 2. Let $F_{1}$ and $F_{2}$ be two product preserving bundle functors on $\mathcal{F}$ ol. The correspondence $\nu \rightarrow \eta^{\nu}$ is a bijection between natural transformations $F_{1} \rightarrow F_{2}$ and morphisms $\mu^{F_{1}} \rightarrow \mu^{F_{2}}$ between corresponding Weil algebra homomorphisms. The inverse correspondence is $\eta \rightarrow \nu^{[\eta]}$.

10. An exchanging isomorphism. Let $F_{1}$ and $F_{2}$ be product preserving bundle functors on $\mathcal{F}$ ol. According to Remark 2 the composition $F_{1} \circ F_{2}$ is again a product preserving bundle functor on $\mathcal{F}$ ol. Let $\mu^{F_{1}}, \mu^{F_{2}}$ and $\mu^{F_{1} \circ F_{2}}$ be the corresponding Weil algebra homomorphisms. Using the tensor product we have the Weil algebra homomorphism $\mu^{F_{1}} \otimes \mu^{F_{2}}$.

It is easy to see that $\left(F_{1} \circ F_{2}\right)_{\mid \mathcal{F M}}=\left(F_{1}\right)_{\mid \mathcal{F} \mathcal{M}} \circ\left(F_{2}\right)_{\mid \mathcal{F M}}$. By the definition $\mu^{F_{1}}$ corresponds to $\left(F_{1}\right)_{\mid \mathcal{F} \mathcal{M}}$, etc. Then by the theory of product preserving bundle functors on $\mathcal{F} \mathcal{M}$ (see [4]) we have

Proposition 3. $\mu^{F_{1} \circ F_{2}}=\mu^{F_{1}} \otimes \mu^{F_{2}}$. 
COROLlaRY 4. We have the isomorphism $F_{1} \circ F_{2} \cong F_{2} \circ F_{1}$ corresponding to the exchanging isomorphism of the tensor product.

\section{References}

[1] M. Doupovec and I. Kolář, On the jets of fibered manifold morphisms, Cah. Topol. Géom. Différ. Catég. 40 (1999), 21-30.

[2] I. Koláŕ et al., Natural Operations in Differential Geometry, Springer, 1993.

[3] W. M. Mikulski, Product preserving bundle functors on fibered manifolds, Arch. Math. (Brno) 32 (1996), 307-316.

[4] W. M. Mikulski and J. M. Tomáš, The natural operators lifting k-projectable vector fields to product preserving bundle functors on $k$-fibered manifolds, Univ. Iagel. Acta Math. 41 (2003), 273-282.

[5] R. A. Wolak, On transverse structures of foliations, Rend. Circ. Mat. Palermo (2) Suppl. 9 (1985), 227-243.

Institute of Mathematics

Jagiellonian University

Reymonta 4

30-059 Kraków, Poland

E-mail: mikulski@im.uj.edu.pl 